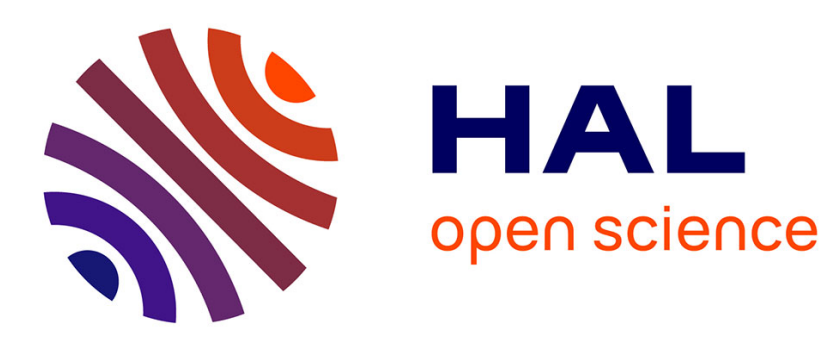

\title{
Compteur multipointe à F.C. appliqué à la détection de l'émission exoélectronique thermostimulée (E.E.T.S.)
}

G. Comby, J. Quidort, J.F. Chalot, G. Valladas, A. Zadra, M. Petel, J. Barthe

\section{To cite this version:}

G. Comby, J. Quidort, J.F. Chalot, G. Valladas, A. Zadra, et al.. Compteur multipointe à F.C. appliqué à la détection de l'émission exoélectronique thermostimulée (E.E.T.S.). Revue de Physique Appliquée, 1983, 18 (4), pp.263-270. 10.1051/rphysap:01983001804026300 . jpa-00245090

\section{HAL Id: jpa-00245090 https://hal.science/jpa-00245090}

Submitted on 1 Jan 1983

HAL is a multi-disciplinary open access archive for the deposit and dissemination of scientific research documents, whether they are published or not. The documents may come from teaching and research institutions in France or abroad, or from public or private research centers.
L'archive ouverte pluridisciplinaire HAL, est destinée au dépôt et à la diffusion de documents scientifiques de niveau recherche, publiés ou non, émanant des établissements d'enseignement et de recherche français ou étrangers, des laboratoires publics ou privés. 


\title{
Compteur multipointe à F.C. appliqué à la détection de l'émission exoélectronique thermostimulée (E.E.T.S.)
}

\author{
G. Comby, J. Quidort, J. F. Chalot, G. Valladas, A. Zadra, M. Petel (*) et J. Barthe (*) \\ Département de Physique des Particules Elémentaires, CEN-Saclay, 91191 Gif-sur-Yvette Cedex, France
}

(Reçu le 12 octobre 1982, révisé le 18 janvier 1983, accepté le 19 janvier 1983)

\begin{abstract}
Résumé. - Les détecteurs multipointes à focalisation cathodique développés pour détecter et localiser des électrons uniques sont tout désignés pour révéler des événements à très faible population d'électrons primaires. Dans les phénomènes d'exo-émission le dépiégeage des centres se manifeste, entre autres choses, par l'émission de 1 à quelques électrons d'énergie voisine de l'électron-volt. Ce travail expose le comportement des compteurs multipointes dans la détection des exoélectrons thermostimulés.
\end{abstract}

Abstract. - The multi-needle detectors with cathode focusing, developed to detect and to localize single photo electrons are specially adapted to reveal events with a very poor number of primary electrons. In exo-emission phenomena each event appears like a emission of 1 to some electrons with a weak energy $(\sim 1 \mathrm{eV})$. This work describes the performances of the multi-needle counters when there are used to detect thermo-stimuled exoelectrons.

1. Introduction. - Certains matériaux préalablement excités émettent un flux d'électrons au cours d'une stimulation thermique. Cette propriété relève du phénomène nommé émission exoélectronique.

En particulier, si l'excitation provient d'une irradiation par un rayonnement ionisant (radioactivité, faisceau de particules, etc...), la population des électrons recueillis lors d'une stimulation thermique est liée à la dose intégrée dans l'échantillon.

Cette technique présente également d'autres particularités qui intéressent les spécialistes de la radioprotection en vue d'une application à la dosimétrie du personnel.

La détection de l'émission exoélectronique thermostimulée (E.E.T.S.) peut s'effectuer selon l'application, à l'aide d'un multiplicateur d'électrons nécessitant un vide de bonne qualité [1,2], par intégration grâce à une chambre d'ionisation, [3], ou par l'emploi d'un compteur. à multiplication électronique dans les gaz [4-8].

Les compteurs à fils fonctionnant en régime GM ou proportionnel, ainsi que les compteurs à pointe ont largement été utilisés et ont permis la compréhension de ce phénomène et le développement de certaines applications.

La mise au point de détecteurs multipointes à foca-

(*) IPSN/DPr/STEPD. lisation cathodique (M.P.F.C.) [10], conçus en vue de détecter et localiser des photoélectrons uniques, doit permettre d'étendre les possibilités et les performances de cette technique.

Cette étude relate le comportement du compteur multipointe à focalisation cathodique directement associé à la détection des exoélectrons sans une adaptation particulière du détecteur aux conditions expérimentales spécifiques imposées par cette technique. Ces observations et ces mesures nous permettent de proposer une première génération de détecteur plus opérationnelle.

2. Appareillage. - L'étude de l'émission exoélectronique thermostimulée nécessite l'association de 5 fonctions pour composer l'appareillage de base. On distingue successivement (schéma Fig. 1) :

- les échantillons ou dépôts émissifs;

- l'excitation des échantillons;

- la stimulation en vue d'une mesure (chauffage);

- la détection des exoélectrons émis;

- l'acquisition et la gestion de la mesure.

- Les échantillons utilisés ici sont des dépôts, sur support métallique ou support de graphite, d'alumine [8], d'oxyde de béryllium [9] obtenus soit par projection d'un plasma [10] soit par évaporation sous vide. Destinées à jouer le rôle de dosimètres, ces pas- 


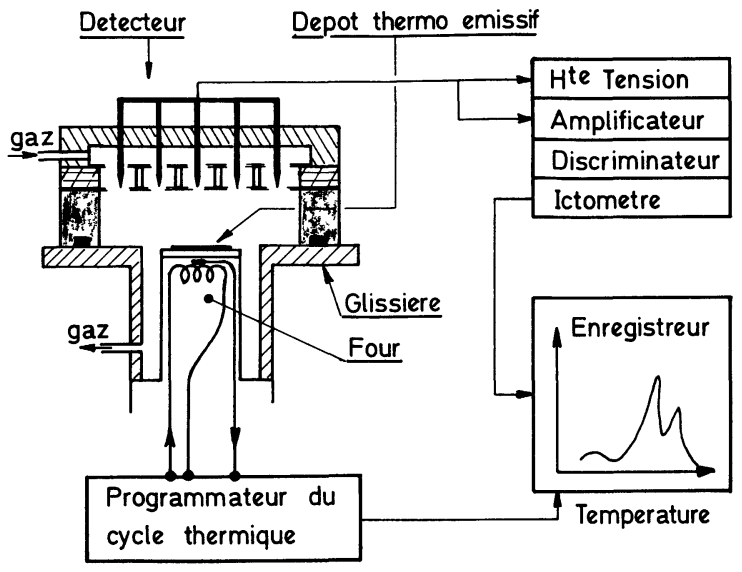

Fig. 1. - Synoptique fonctionnel d'un dispositif de mesure de l'émission exoélectronique thermostimulée.

[Functional diagram of an apparatus to measure thermostimulated exo-electronic emission.]

tilles font l'objet d'un développement particulier et sont élaborées avec le plus grand soin en vue de l'homogénéité de la réponse à une même excitation [8-10].

- L'excitation des échantillons a volontairement été limitée à l'irradiation $\beta$ d'une source de ${ }^{90} \mathrm{Sr}$. Un montage mécanique simple permet des conditions d'irradiation reproductibles (distance, angle solide, temps d'exposition, etc...).

- La stimulation thermique s'effectue par l'intermédiaire d'un four sur lequel est fixée la pastille. Un programmateur régule l'alimentation électrique en fonction du signal délivré par le thermo-couple appliqué contre la pastille et des informations affichées définissant le cycle de chauffe désiré.

- La détection des exoélectrons, émis avec une énergie de l'ordre de l'électron-volt, nécessite l'introduction de la surface émissive dans le volume sensible du compteur. Au paragraphe suivant, nous décrivons la structure mécanique et électrostatique du détecteur, son principe de fonctionnement et l'électronique associée.

\subsection{STRUCTURE MÉCANIQUE ET ÉLECTROSTATIQUE. -} La figure 2 regroupe un aspect de la conception mécanique du compteur, et un aspect de la distribution électrostatique des lignes de champ électrique correspondantes, qui couplent à travers le diaphragme, les volumes sensibles des cellules à la surface exo-émissive.

Une étude plus complète de ces compteurs est relatée dans l'article [11].

Dans le présent montage nous distinguons :

- la zone exo-émissive, maintenue en contact avec le four et donc mise à la masse ;

- un espace de dérive qui sépare la zone émissive du plan cathodique;

- le plan cathodique : fine feuille de cuivre de 30 à $50 \mu \mathrm{m}$ d'épaisseur percée de trous dans l'axe desquelles se placent les aiguilles;

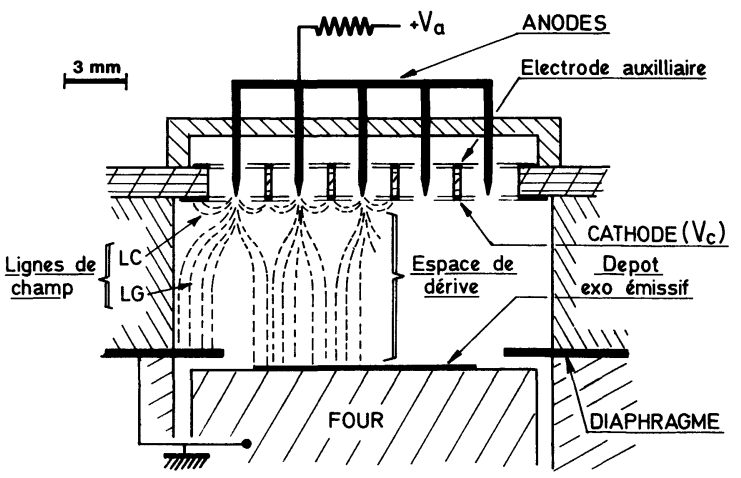

Fig. 2. - Coupe schématique du détecteur MPFC et du four. Les pointillés issus des anodes simulent les lignes de champ électrique (LG) qui drainent les exoélectrons vers les zones multiplicatrices.

[Schematic cross-section of the MPFC detector and oven. Dotted lines issuing from the anodes indicate the electric field lines (LG) which drift the exo-electrons toward the zones of multiplication.]

— le plan "électrode auxiliaire " - réplique du plan cathodique - porté au même potentiel que les aiguilles;

- les pointes ou anodes portées à un potentiel positif, à l'extrémité desquelles se produit la multiplication électronique.

La cathode peut être portée à un potentiel différent de celui de la masse. Les lignes de champ électrique LG drainent les exoélectrons vers les zones multiplicatrices.

Le four et l'échantillon présentant une symétrie de révolution, nous ont incité à conserver cette symétrie dans l'interconnection des anodes. La surface émissive est donc observée par trois zones concentriques indépendantes (centrale : 7 aiguilles, intermédiaire $: 12$ aiguilles, externe $: 18$ aiguilles) qui peuvent être à leur tour interconnectées pour attaquer le même canal électronique.

Lors des mesures le détecteur est vertical, les pointes des aiguilles dirigées vers le bas. La circulation continue du méthane renouvelle constamment le gaz au niveau des zones multiplicatrices et entraîne le gaz chaud et éventuellement pollué vers la sortie.

Aucun refroidissement du corps du compteur, autre que la diffusion thermique entre les pièces en contact n'est effectué dans ces tests.

\subsection{Excitation DU COMPTEUR. - Afin d'observer} le comportement du détecteur dans des conditions expérimentales particulières, nous avons utilisé divers types d'excitation pour faire apparaître dans le volume sensible les électrons nécessaires à la détection des événements.

Une glissière à 3 positions permet de disposer successivement dans le compteur :

- des sources radioactives;

- le four équipé du dépôt exo-émissif;

- un hublot de quartz. 
Grâce à ce hublot une insolation aux ultra-violets $(\lambda \sim 250 \mathrm{~nm})$ crée des événements à un seul électron par effet photo-électrique sur la cathode.

L'emploi d'une source $\gamma-$ ici source de ${ }^{22} \mathrm{Na}$ blindée émettrice $\gamma$ de $511 \mathrm{keV}$ - permet d'exciter le compteur lors d'un cycle de température.

2.3 FONCTIONNEMENT DU COMPTEUR. - Le schéma électrostatique proposé figure 2 nous aide à dissocier les différentes phases qui conduisent à la détection des événements.

L'émission exoélectronique procède d'un phénomène physique que nous n'aborderons pas ici. Nous le caractériserons pour l'instant par l'émission dans un temps court et une surface limitée d'un ou plusieurs électrons d'énergie cinétique très faible $(\sim 1 \mathrm{eV})$. Ces paramètres d'espace et de temps (et bien d'autres) dépendent des conditions expérimentales, qui font l'objet d'un autre travail.

Emis hors du dépôt, ces électrons libres sont alors drainés par les lignes de champ électriques issues de la pointe des aiguilles. Ces lignes de force assurent un couplage électrostatique entre des zones jointives du dépôt émetteur et les pointes détectrices. Selon la précision envisagée cette " régionalisation » est une localisation des phénomènes.

Arrivés à une centaine de microns de l'extrémité de l'aiguille, la multiplication par chocs des électrons sur les molécules du gaz déclenche un régime d'avalanches créant un flot d'électrons sur les anodes et une calotte ionique positive près de la pointe qui provoque une coupure électrostatique du phénomène.

La migration de ces ions entraîne un temps mort à l'image de la dimension des cellules multiplicatrices. L'étude [12] révèle des temps morts de quelques $\mu s$ dépendant du mélange gazeux, de la géométrie des cellules, du seuil de l'électronique associée.

Bien que ces compteurs fonctionnent avec une grande diversité de mélanges gazeux et vapeurs pures, les tests relatés ici ont été effectués exclusivement en méthane pur.

2.4 EleCtronique D'ACQUiSition. - Compte tenu du développement rapide de la décharge au niveau de la pointe et du regroupement des aiguilles sur la même voie électronique nous avons développé un amplificateur et une mise en forme rapide des signaux en regard du temps mort d'une cellule, figure 3 .

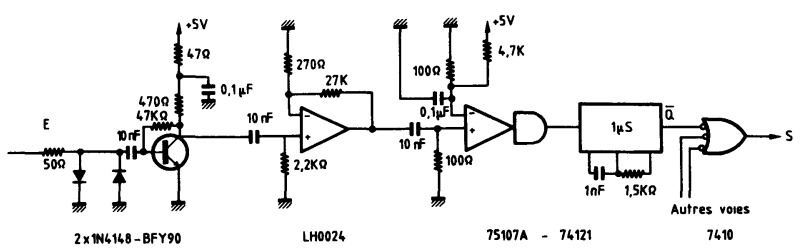

Fig. 3. - Circuit électronique d'amplification et de mise en forme des signaux.

[Electronic circuit for amplification and shaping of the signals.]
La largeur des signaux de sortie $>200$ ns est imposée par l'ictomètre au standard NIM. Une table traçante $X, Y$ enregistre l'évolution du taux de comptage en fonction de la température. Des échelles effectuent la sommation des impulsions recueillies au cours de la stimulation thermique.

3. Observation du comportement du détecteur multipointes. - 3.1 CARACTÉRISTIQUe dE COMPTAGE. - En atmosphère de méthane pur, à la température du laboratoire $\left(20^{\circ} \mathrm{C}\right)$ la courbe $a$ figure 4 représente la variation du taux de comptage en fonction de la tension anode quand une source $\beta$ de ${ }^{14} \mathrm{C}$ est introduite dans le compteur. Compte tenu du parcours $\operatorname{des} \beta$, l'ionisation se trouve distribuée au même instant dans les volumes sensibles de plusieurs cellules. La sommation des impulsions délivrées en phase par ces cellules attaque l'électronique de comptage. Ceci explique la faible tension de départ du plateau comparativement à d'autres sources d'ionisation, ainsi que la dynamique en amplitude des impulsions recueillies.

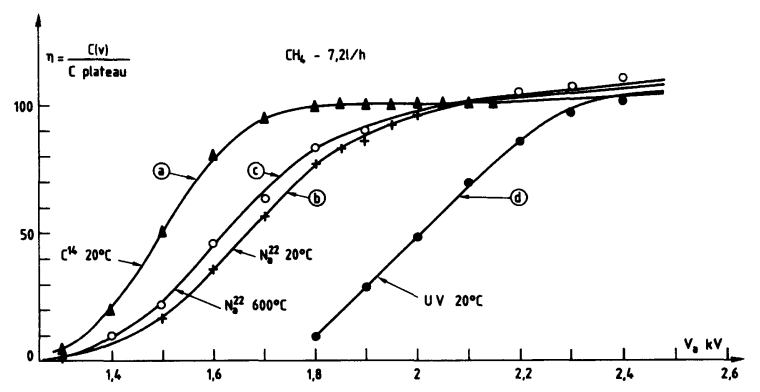

Fig. 4. - Caractéristiques de comptage normalisée : a) source : ${ }^{14} \mathrm{C}, T$ du four : $20^{\circ} \mathrm{C} ; b$ ) source : ${ }^{22} \mathrm{Na}$ (blindée), $T$ du four : $20^{\circ} \mathrm{C} ; c$ ) source : ${ }^{22} \mathrm{Na}$ (blindée), $T$ du four : $\left.600{ }^{\circ} \mathrm{C} ; d\right)$ source : Ultra-violet, $T$ du four : $20^{\circ} \mathrm{C}$. La montée en température du four ne perturbe pas le fonctionnement $\mathrm{du}$ compteur.

[Normalized counting rates $: a) ; b$ ) $; c$ ) $; d$ ). The performance of the counter is not perturbed by the increase in temperature.]

La courbe $b$ de la même figure, obtenue sous irradiation externe d'une source de ${ }^{60} \mathrm{Co}$ qui, en moyenne, laisse moins d'ionisation par interaction dans le compteur que les $\beta$ précédents, montre une montée au palier plus lente.

Dans les mêmes conditions d'irradiation, le relevé du palier, alors que le four est maintenu à $600^{\circ} \mathrm{C}$ (courbe $c$ ) ne révèle aucun trouble de fonctionnement.

En découplant ainsi l'origine de l'ionisation des conditions de détection ce test montre la stabilité de fonctionnement du compteur traversé par un flux de méthane de $6 \mathrm{l} / \mathrm{h}$ lorsque le four est maintenu à température constante.

Bien que le corps du compteur soit réalisé en verre époxy, le dégazage de ce matériau sous l'action de la chaleur ne perturbe pas de façon appréciable le développement des avalanches et par conséquent la détection lors du cycle thermique. 
Afin de repérer le domaine de sensibilité capable de détecter les photoélectrons uniques on observera la caractéristique de comptage obtenue sous insolation U.V. : courbe $d$. Un palier est atteint à partir de $2300 \mathrm{~V}$.

Si donc, pour certain dépôt exo-émetteur, le dépiégeage s'effectue en n'éjectant qu'un seul électron, les conditions de détection offertes par ce compteur permettront leur mise en évidence pour des tensions d'anodes comprises entre 2300 et $2600 \mathrm{~V}$.

L'analyse statistique de 59 comptages consécutifs (tension d'anode : $2000 \mathrm{~V}$ - source ${ }^{14} \mathrm{C}$ - temps de comptage : $10 \mathrm{~s}$ - atmosphère de méthane) donne un rapport (Ecart type/moyenne) de 2,6 $\times 10^{-3}$ traduisant une excellente homogénéité des mesures.

3. 2 DÉTECTION DES THERMO EXó́LECTRONS. - Dans ces conditions à une tension anode de $2400 \mathrm{~V}$ les courbes $a$ et $b$ de la figure 5 représentent les variations des taux de comptage recueillis quand on accroît

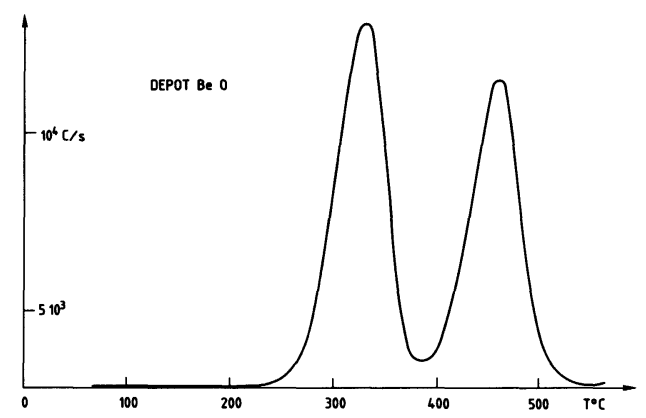

a)

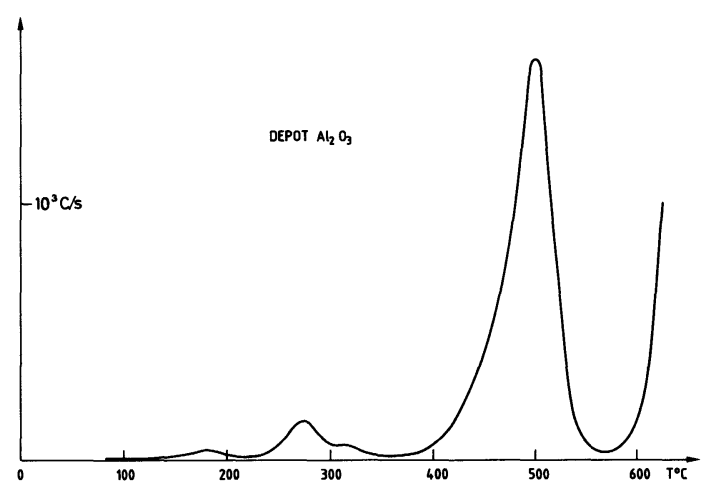

b)

Fig. 5. - Caractéristiques de thermostimulation : a) d'un dépôt de $\mathrm{BeO} ; b$ ) d'un dépôt de $\mathrm{Al}_{2} \mathrm{O}_{3}$.

(Characteristics of thermo-stimulation for a deposit of $\mathrm{BeO}(a)$ and $\mathrm{Al}_{2} \mathrm{O}_{3}(b)$.].

linéairement la température des deux dépôts exoémissifs $\left(\mathrm{Al}_{2} \mathrm{O}_{3}\right.$ et $\left.\mathrm{BeO}\right)$ préalablement irradiés par le rayonnement d'une source $\beta$ de ${ }^{90} \mathrm{Sr}$. Pour une même excitation et des stimulations identiques, la figure 6 reproduit la caractéristique de comptage en exoélectrons. Entre 2200 et $2500 \mathrm{~V}$ un palier est

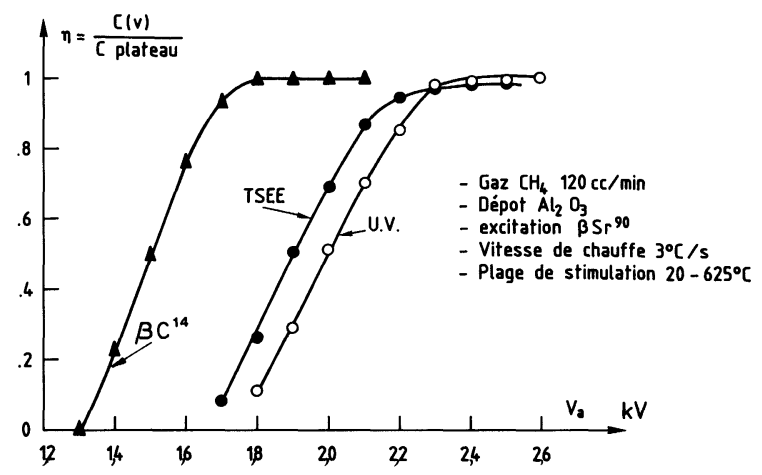

Fig. 6. - Représentation de la caractéristique de comptage observée en TSEE par rapport à celles relevées avec une source $\beta\left({ }^{14} \mathrm{C}\right)$ ou sous insolation UV $(250 \mathrm{~nm})$.

[Normalized counting rates observed for TSEE compared to those obtained with a $\beta$ source $\left({ }^{14} \mathrm{C}\right)$ and with a UV light source $(250 \mathrm{~nm})$.]

atteint qui débute avant le plateau observé sous insolation U.V.

Pour une tension anodique de $2400 \mathrm{~V}, 10$ mesures successives effectuées sur le même dépôt d'oxyde de béryllium dans des conditions de reproductibilité strictement contrôlées donnent un rapport (écart type/moyenne des mesures) de $3 \%$.

L'indépendance des cellules a déjà été vérifiée à l'aide de photoélectrons obtenus sous insolation U.V. (Réf. [10]). Nous avons reproduit ce test en émission exoélectronique thermostimulée (E.E.T.S.) en déplaçant à l'aplomb des cellules une surface émissive diaphragmée par un trou de $3 \mathrm{~mm}$ de diamètre.

Les anodes sont regroupées en 3 zones concentriques reliées chacune à une voie de comptage afin d'observer les comportements et les influences des cellules entre elles au cours d'un cycle thermique.

La figure 7 reproduit l'évolution des comptages des 3 zones au cours de ce déplacement. Un maximum est observé quand l'axe du diaphragme coïncide avec l'axe d'une cellule. Les variations observées entre deux axes sont dues à la modification de la distribution des lignes de champ, qui, de façon préférentielle vont relier les anodes à la zone pleine du diaphragme.

Avec une ouverture plus importante, le couplage s'uniformise et établit une collection homogène des charges. Ces résultats nous indiquent que même à température élevée les zones détectrices en fonctionnement n'engendrent pas de comptage parasite dans les zones voisines.

Cette observation souligne à la fois la possibilité d'utiliser ces compteurs pour réaliser une cartographie des surfaces émettrices avec la précision du pas des cellules et celle d'accroître les performances de détection en sommant les signaux comptabilisés sur chaque anode.

Afin d'éprouver la dynamique de détection du compteur nous avons soumis le dépôt de $\mathrm{BeO}$ à des doses de plus en plus intenses. Avec une vitesse de 


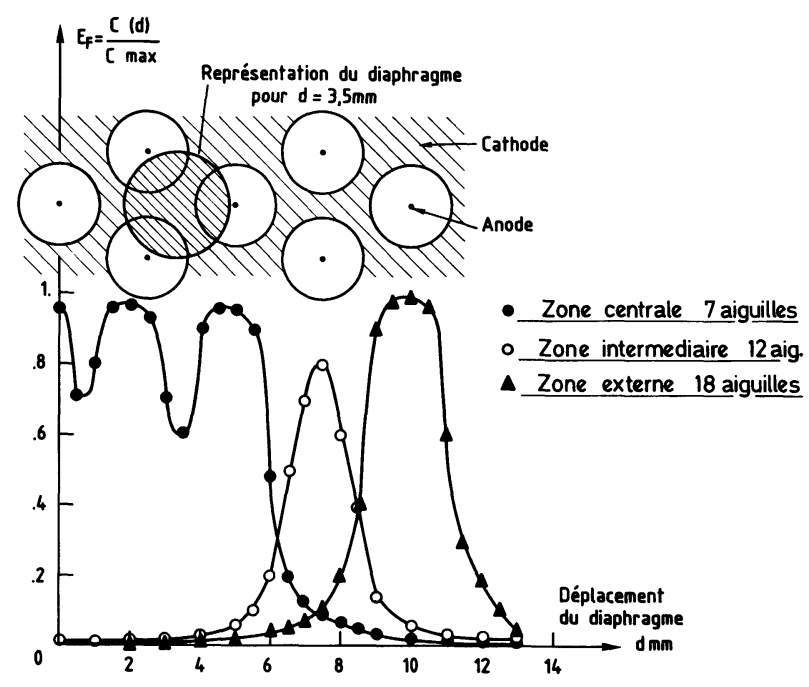

Fig. 7. - Variation du comptage total observé simultanément sur les trois groupements concentriques d'anodes quand on déplace sous le compteur un dépôt TSEE diaphragmé. Ces courbes révèlent l'indépendance des cellules détectrices.

[Variation of the total counting observed simultaneously for the three concentric groups of anodes when the source (TSEE deposit with diaphram) is moved across the counter. These curves show that the detector cells count independently.]

chauffe de $2^{\circ} \mathrm{C} / \mathrm{s}$ entre 150 et $425^{\circ} \mathrm{C}$ la figure 8 montre une bonne linéarité jusqu'à des taux de comptage de $4,5 \times 10^{5} \mathrm{c} / \mathrm{s}$, les 37 aiguilles étant interconnectées sur la même voie de comptage. L'observation du palier en E.E.T.S. de la figure 6 obtenue pour des taux

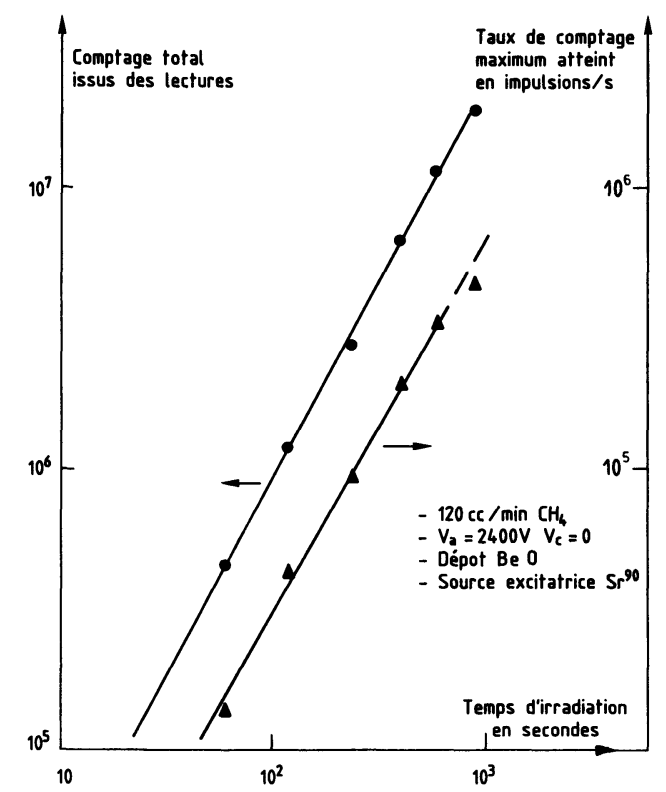

Fig. 8. - Evolution du taux de comptage maximum et du comptage total en fonction de la dose induité dans un dépôt de $\mathrm{BeO}$.

[Maximum counting rate and total counts observed as a fonction of the radiation dose received by the $\mathrm{BeO}$ deposit.] de comptage beaucoup plus faibles indique bien une détection totale des phénomènes ionisants libérés lors du chauffage et non un effet de saturation dans le fonctionnement du compteur. Ici la taille des cellules réduit le temps mort à quelques $\mu$ s ce qui explique des taux de comptages atteints ici comparés aux performances d'un compteur GM classique.

Individualiser l'acquisition des signaux au niveau de chaque pointe permet d'augmenter dans de larges proportions la dynamique d'analyse du détecteur.

3. 3 Mouvement PROPRE. BRUIT DE FOND. - A la température du laboratoire le compteur présente, en fin de palier un mouvement propre de l'ordre de $0,5 \mathrm{c} / \mathrm{s}$ pour l'ensemble des 37 aiguilles.

Le comptage enregistré, lors d'une stimulation de l'échantillon n'ayant pas été soumis à une excitation, constitue le bruit de fond de la mesure.

Les chauffes successives d'un dépôt d'alumine sur un disque d'acier inoxydable, à raison de $2^{\circ} \mathrm{C} / \mathrm{s}$ jusqu'à $650^{\circ} \mathrm{C}$ font apparaître une décroissance des comptages jusqu'à obtenir un plateau : figure 9 .

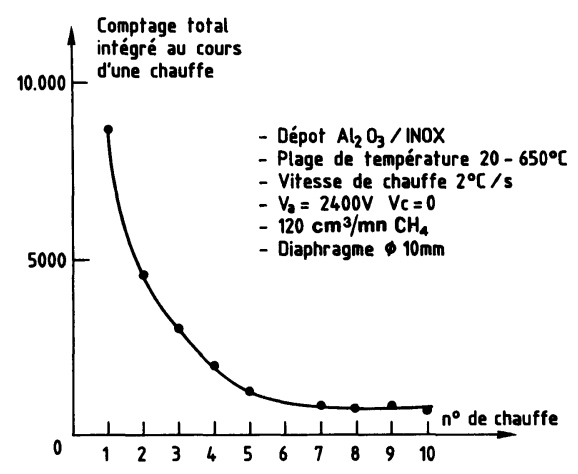

Fig. 9. - Décroissance du bruit de fond observé lors des cycles thermiques successifs sans irradiation du dépôt.

[Decrease of background counts observed for successive temperature cycles with no new irradiation of the deposit.]

Le comptage recueilli de l'ambiante jusqu'à $600^{\circ} \mathrm{C}$ ne représente que 10 à $20 \%$ du comptage final à $650^{\circ} \mathrm{C}$. Lors de la première mise en route d'un compteur, les premières stimulations en température ont pour effet d'améliorer le bruit de fond de la mesure.

Afin d'atteindre un bruit de fond aussi faible que possible les matériaux les moins thermoémissifs doivent être choisis pour constituer l'appareillage de détection. La figure 10 présente les activités enregistrées avec des disques de matériaux divers soumis aux mêmes conditions de chauffe que les pastilles supportant les dépôts émissifs.

Bien que tous ces disques ne soient pas de même épaisseur, ces enregistrements situent comparativement au dépôt d'alumine, les débuts des domaines d'émission thermo-ionique de ces substances. Ils montrent en outre, la stabilité de fonctionnement du compteur dans une très large gamme de température $\left(>650^{\circ} \mathrm{C}\right)$. Ceci permet d'attribuer respectivement 


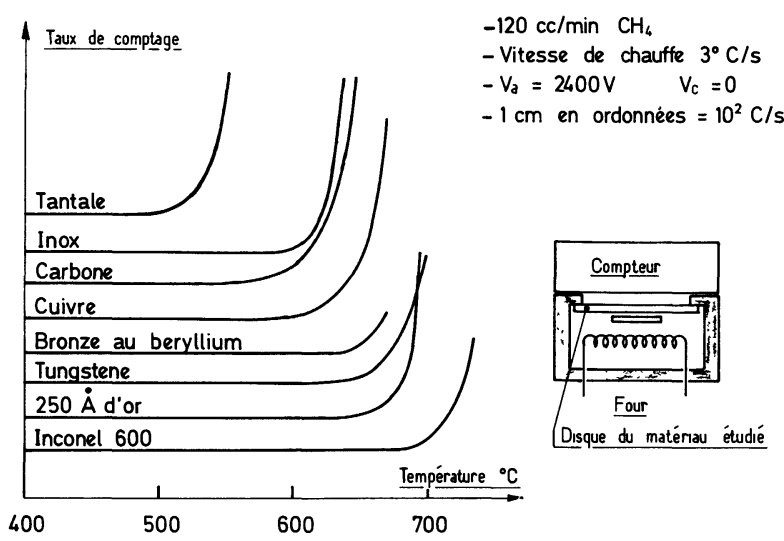

Fig. 10. - Caractéristique de thermo-émission de divers matériaux.

[Thermo-emission caracteristics for different materials.]

au compteur et au dépôt sa propre participation au bruit de fond d'une mesure.

3.4 ACTION DU CHAMP ÉLECTRIQUE DE DRAINAGE. La collection des exoélectrons s'effectue par les lignes de champ LG de la figure 2 établies pour une tension cathode nulle. En translatant les tensions anode et cathode de telle sorte que $\left(V_{\mathrm{a}}-V_{\mathrm{c}}\right)$ reste constant, on maintient de façon stable les conditions de multiplication électronique et on agit sur l'intensité et sur l'implantation des lignes de force qui couplent l'échantillon aux zones multiplicatrices.

La figure 11 montre l'influence d'un changement de la polarisation de la cathode sur le comptage total recueilli lors des cycles de mesure. Les conditions

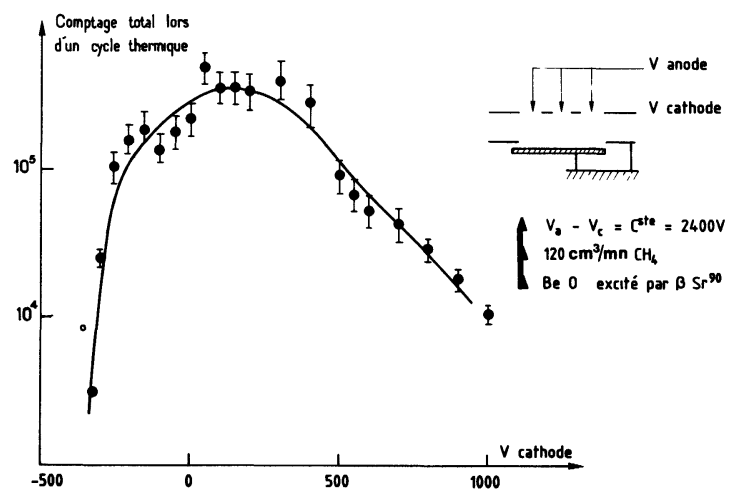

Fig. 11. - Influence du potentiel cathode $V_{c}$ sur le comptage total pour une différence constante du potentiel anodecathode $\left(V_{\mathrm{a}}-V_{\mathrm{c}}\right)$.

[Influence of the cathode voltage $V_{c}$ on the total count observed for constant anode-cathode potential difference $\left.\left(V_{\mathrm{a}}-V_{\mathrm{c}}\right) \cdot\right]$

optimums sont observées pour une tension cathode $V_{\mathrm{c}}$ égale à $10 \%$ de la tension anode appliquée. Ce résultat est en accord avec les mesures d'efficacité de collection des électrons provenant de la photo-ionisation du mélange gazeux dans l'espace de dérive [10]. Durant ces essais, nous n'avons remarqué aucune modifications significatives de l'émission électronique en fonction de la température.

3.5 INCIDENCE DU REMPlisSAGE. - Comme l'indique la figure 1, la présentation de l'échantillon ou des autres sources d'ionisation dans le compteur s'effectue au moyen d'une glissière. A chaque manouvre, nous introduisons une quantité d'air représentant 10 à $50 \%$ du volume sensible du compteur. Compte tenu du sens de la circulation gazeuse et de la densité du méthane par rapport à l'air, la composition de l'atmosphère gazeuse dans les zones de multiplication n'est pas affectée de façon significative par ces manœuvres. Les tests ont montré que même avec un débit de $31 / \mathrm{h}$ aucun rinçage n'était nécessaire avant de lancer la mesure de l'activité d'une source $\beta$.

Par contre la population des exoélectrons peut être décimée par attachement électronique à des molécules de gaz polluant le méthane.

En effet, outre son action sur l'intensité du champ électrique, l'augmentation de la hauteur de l'espace de dérive allonge les parcours des exoélectrons pour atteindre les zones de multiplication. Selon la pollution en gaz électronégatif de l'atmosphère gazeuse du compteur (oxygène de l'air par exemple), la capture des électrons par attachement électronique pour former un ion négatif peut devenir gênante. C'est précisément à l'interface compteur-glissière que se situe l'échantillon exo-émetteur. Bien que cette pollution ne soit pas ressentie au niveau de la multiplication électronique, elle est une cause potentielle des fluctuations des mesures par action sur la collection des électrons.

Pour illustrer son incidence nous rappellerons (réf. [13]) qu'une atmosphère de méthane polluée à $10 \%$ en oxygène élimine $10 \%$ des électrons initiaux par cm de parcours. Pour des événements correspondant à l'émission d'un seul électron, l'efficacité de détection est directement réduite dans la même proportion.

En conséquence la technologie d'un compteur destiné à une exploitation intensive doit prévenir toute évolution de la nature du remplissage, afin d'obtenir des mesures statistiquement reproductibles.

3.6 Dimensionnement DU COMPTEUR. - Plus l'espace de dérive est grand, plus il éloigne la source de rayonnement infra-rouge des zones de multiplication. Ceci réduit les effets secondaires tels que thermodissociation, dégazage, effet photoélectrique, déformations etc... qui interviennent soit au niveau de la détection soit pour accroître le bruit de fond.

Ceci affaiblit le champ électronique de drainage que l'on peut compenser par une polarisation de la cathode par rapport à l'échantillon. De ce fait cette dimension ne joue pas un rôle déterminant dans la conception du corps du compteur.

Une étude [12] a montré qu'à la température du 
laboratoire une réduction d'un facteur 2 de la taille et du pas des cellules conduit encore à un détecteur opérationnel.

La taille, la répartition et le nombre des cellules, pour des espacements entre pointes supérieures à $1,5 \mathrm{~mm}$, relèvent essentiellement des exigences expérimentales.

\subsection{COMPORTEMENT SELON LES VITESSES DE CHAUFFE.} Nous avons soumis un même dépôt d'alumine à des cycles de mesure où la reproductibilité de l'excitation et celle de la détection restaient identiques. Seule la vitesse de chauffe allait croissant d'un cycle à l'autre. La figure 12 reproduit deux enregistrements obtenus à $1^{\circ} \mathrm{C} / \mathrm{S}$ et $4^{\circ} \mathrm{C} / \mathrm{S}$ avec une alumine. Nous remarquons une translation en température du pic - qui résulte, en grande partie, de l'inertie du dispositif de stimulation thermique - et une stabilité des comptages sommés et des largeurs à mi-hauteur des pics.

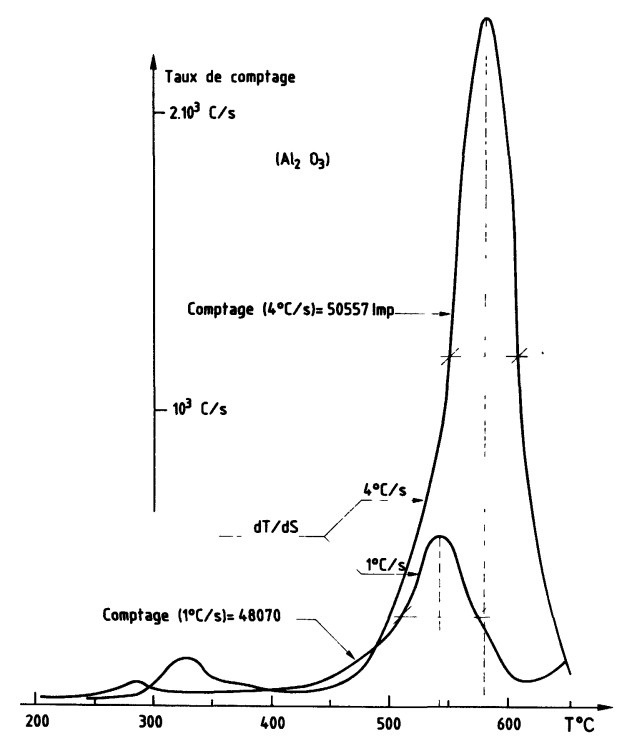

Fig. 12. - Influence de la vitesse de chauffe sur la caractéristique de thermo-stimulation d'un même dépôt d'alumine.

[Influence of the heating rate on the TSEE response for the same $\mathrm{Al}_{2} \mathrm{O}_{3}$ deposit.]

Ceci nous permet de définir la dynamique d'utilisation de l'appareillage allant de très fortes doses d'excitation nécessitant une très lente stimulation, aux très faibles doses révélées par des chauffes rapides. Le taux de comptage

$$
(\delta N / \delta S)_{\max }=(\delta N / \delta T)(\delta T / \delta S)=\mathrm{Cte} \sim 5 \times 10^{5} \mathrm{I} / \mathrm{S},
$$

s'exprime en fonction du nombre de dépiégeages par unité de température multiplié par la vitesse de chauffe. Les conditions limites de mesure sont imposées par le taux de comptage maximum $(\delta N / \delta S)$ et la stimulation thermique maximum $(\delta T / \delta S)$.

Sur le diagramme de la figure 13, la dynamique de lecture des échantillons se situe entre la branche

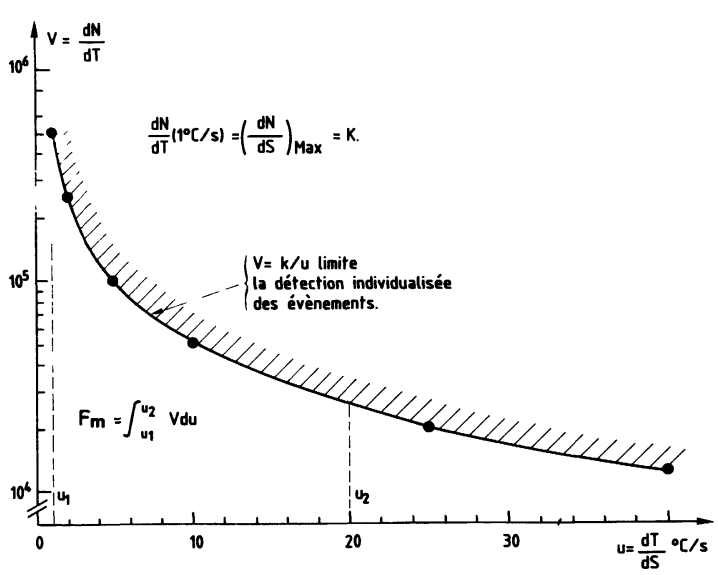

Fig. 13. - Visualisation du domaine de détection « individualisée " des exoélectrons pour un appareillage de lecture TSEE donné. Cette représentation associe les performances du détecteur et du thermo-stimulateur.

[This diagram shows the "individualized " detection field of the exoelectrons for a given apparatus. This diagram associates the detector and thermo-stimulator performances.]

d'hyperbole et les axes des coordonnées. Par ailleurs, $(\delta N / \delta T)$ est une fonction croissante de l'intensité de l'excitation du dépôt c'est-à-dire ici de la dose intégrée. Pour chaque dose, il existe donc une stimulation limite au-delà de laquelle les électrons sont émis de façon quasi continue, imposant au détecteur non plus des événements individualisés dans le temps, mais l'équivalent d'un plasma provoquant l'étouffement du compteur.

Ce diagramme permet en outre de classer selon leur dynamique de mesure les ensembles de détection en associant les trois principales fonctions :

- les caractéristiques du dépôt $(\mathrm{d} N / \mathrm{d} T)=V$;

- la capacité de la stimulation $(\mathrm{d} T / \mathrm{d} S)=U$;

- les performances du détecteur $(\mathrm{d} N / \mathrm{d} S)=$ $k=4,5 \times 10^{5}$.

L'aire comprise entre la branche d'hyperbole, l'axe des abscisses et les conditions limites de chauffe peut être interprétée comme un facteur de mérite $F_{\mathrm{m}}$ de l'appareillage :

$$
F_{\mathrm{m}}=\int_{U_{1}}^{U_{2}} \frac{k}{u} \mathrm{~d} u=k \log U \mid \begin{aligned}
& U_{1} \\
& U_{2}
\end{aligned} .
$$

En prenant $U_{1}=1{ }^{\circ} \mathrm{C} / \mathrm{s}, F_{\mathrm{m}}=k \log \left(U_{2}\right)$.

Pour l'appareillage utilisé ici où $(\delta T / \delta S)_{\max }=$ $U_{2}=20^{\circ} \mathrm{C} / \mathrm{s}$, le facteur de mérite atteint : $F_{\mathrm{m}}=$ $13,48 \times 10^{5}$.

Pour conserver un même facteur de mérite, les performances de la détection doivent augmenter dans les mêmes proportions que les vitesses de chauffe.

L'indépendance des cellules détectrices rend possible une acquisition individualisée sur chaque anode, et, par là, accroît d'un facteur proche du nombre de cellules la dynamique globale de détection. 
4. Conclusion. - Ces quelques résultats ont pour but de montrer que les détecteurs multipointes à focalisation cathodique sont adaptables très simplement à la détection de l'émission exoélectronique thermostimulée(E.E.T.S.).

Le prototype utilisé ici résulte d'une fabrication artisanale de laboratoire et ne fait intervenir aucun matériau sélectionné.

Néanmoins, son emploi fait ressortir certaines caractéristiques, qui peuvent paraître avantageuses en vue d'une exploitation intensive.

Nous citerons seulement :

- la simplicité de fabrication;

- la valeur modérée de la haute tension;

- la robustesse réduisant la maintenance à un simple nettoyage (dans le cas d'analyse de dépôts pulvérulants);
- la large dynamique de comptage;

- la faible sensibilité vis-à-vis de la pollution du mélange gazeux;

- la possibilité d'obtenir de grande surface détectrice capable de prendre en compte la cartographie soit d'un grand dépôt émetteur, soit de nombreux petits dépôts individuels regroupés.

Afin d'optimiser la détection des événements en exoémission, des études complémentaires s'imposent tant dans la détermination de la nature des événements à révéler que dans l'influence des conditions de détection sur les phénomènes exoémissifs.

Remerciements. - Nous remercions Mr Portal (STEPD, CEN/FAR) et Mr Prugne (STIPE, Saclay). Pour les conditions favorables au déroulement de ces essais qu'ils ont su créer dans le cadre de cette collaboration.

\section{Bibliographie}

[1] OnsgaArD, J. and Hougs, E., Int. J. Appl. Radiat. Isot. 24 (1973) 55 (chaneltron).

[2] Braunlich, P., J. Appl. Phys. 42 (1971) 465 (galette micro canaux).

[3] Kramer, J., Proc. Sec. Int. Conf. luminescence Dosimetry, CONF-680920 (Gatlinburg) 1968180.

[4] Kramer, J., Z. Phys. 129 S (1951) 34-44.

[5] KRIKs, H. J., These, Technische Universität, Berlin. Rapport PTB-FMRB-48 (1973).

[6] Eckart, A., Hartung, J., Tagungsberg, Phys. Ges., DDR (1972).

[7] Holzapfel, G., These D.83, Technische Universität, Berlin (1968).
[8] Petel, M., Rapport CEA-R4754 (1976).

[9] Kriegseis, Scharmann, A., Weiss, A., Proc. VIth Internat. Symp. on Exoelectron. Emission and Application, 149-151, Ahrenshoop (RDA), 1979.

[10] Petel, M., Holzapfel, G., Proc. VIth Internat. Symp. on Exoelectron. Emission and Application, 169-170, Ahrenshoop (RDA) 1979.

[11] CombY, G. et al., Nucl. Instrum. Methods 174 (1980) 77.

[12] Сомву, G. et al., Revue Phys. Appl. 16 (1981) 539.

[13] LOEB, L. B., Basic Processes of Gaseous Electronics (Cambridge University Press, London). 